\title{
AN OPTIMISATION-BASED DECISION SUPPORT SYSTEM FRAMEWORK FOR MULTI-OBJECTIVE IN- CORE FUEL MANAGEMENT OF NUCLEAR REACTOR CORES
}

\author{
E.B. Schlünz ${ }^{1,2,3 \#^{*}}$, P.M. Bokov ${ }^{1}$ \& J.H. van Vuuren ${ }^{3}$
}

\section{ARTICLE INFO}

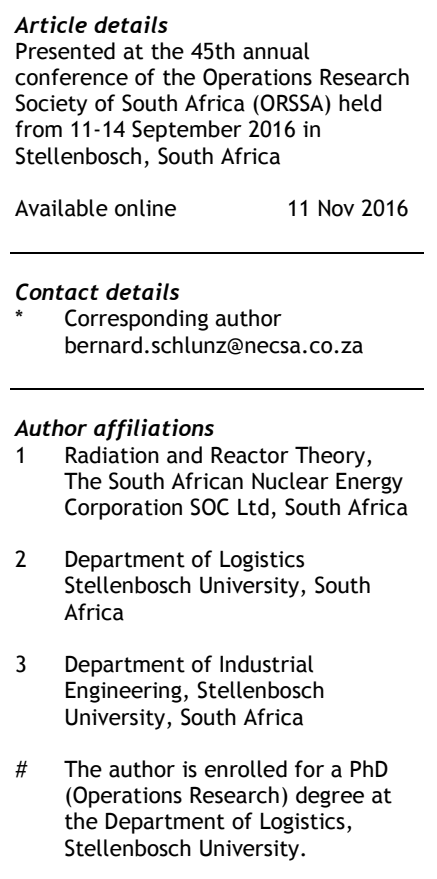

DOI

http://dx.doi.org/10.7166/27-3-1650

\section{ABSTRACT}

The notion of in-core fuel management (ICFM) involves decision making in respect of the specific arrangement of fuel assemblies in a nuclear reactor core. This arrangement, referred to as a reload configuration, influences the efficiency and effectiveness of fuel usage in a reactor. A decision support system (DSS) may assist nuclear reactor operators in improving the quality of their reload configuration designs. In this paper, a generic optimisation-based DSS framework is proposed for multi-objective ICFM, with the intention of serving as a high-level formalisation of a computerised tool that can assist reactor operators in their complex ICFM decisions.

\section{OPSOMMING}

Binne-kern brandstofbestuur (BKBB) behels die neem van besluite oor die rangskikking van brandstofelemente in ' $n$ kernreaktor. So ' $n$ rangskikking staan bekend as ' $n$ herlaai-konfigurasie en beïnvloed die doeltreffendheid en doelmatigheid van brandstofverbruik in die reaktor. ' $n$ Besluitsteunstelsel (BSS) mag kernreaktor-operateurs help om die kwaliteit van hul herlaaikonfigurasie-ontwerpe te verbeter. In hierdie artikel word ' $n$ generiese optimeringsgebaseerde BSS vir veeldoelige BKBB voorgestel wat ten doel het om as ' $n$ hoë-vlak formalisering van ' $n$ gerekenariseerde toepassing te dien wat operateurs kan bystaan met hul komplekse BKBB-verwante besluite.

\section{INTRODUCTION}

During the operation of a nuclear reactor, depleted fuel assemblies are periodically replaced with fresh ones in order to sustain the fission chain reaction occurring in the reactor core. The efficiency and effectiveness of fuel usage in a reactor is influenced by the specific arrangement of fuel assemblies in the core for a given operational cycle. This arrangement is referred to as a reload configuration, and the decision making involved during its design is known as in-core fuel management (ICFM). Finding good reload configurations is a difficult task for a nuclear reactor operator in view of the characteristics associated with ICFM. These include a large combinatorial decision space, multiple nonlinear objectives to pursue, a variety of constraints that have to be satisfied, and significant computational complexity [1]. However, a computerised decision support system (DSS) may assist reactor operators in improving the quality of their reload configuration designs.

ICFM has been studied for several decades [2-6], with the majority of research orientated towards power reactors and within the context of single-objective optimisation. Based on these studies, several computerised tools that may be regarded as DSSs have been developed in academia and industry [7-9]. 
A prominent knowledge-based DSS (or expert system) for ICFM called FUELCON was developed over a period of more than a decade, beginning in the late 1980s [10]. The FUELCON system contains a knowledge base in the form of 'IF-THEN' rule sets. These rule sets are employed within an exhaustive enumeration search mechanism in order to identify families of potential reload configurations. Since the system is interactive, rule sets may be expanded or refined according to the feedback that a decision maker/analyst receives. This manual revision of rule sets may also be performed by an automated procedure that employs neural network learning algorithms [11].

In several optimisation-based DSSs, metaheuristic solution techniques are employed to solve the ICFM optimisation problem. Examples of systems in which simulated annealing is employed are the FORMOSA suite of codes [12], the XIMAGE/SIMAN graphical fuel management and loading pattern optimisation suite [13], and the ROSA software package [9]. Similarly, examples of systems in which a genetic algorithm is employed as the metaheuristic are the CIGARO system [14] and the more extensive GARCO package [7]. These DSS tools have several common features: a variety of choice in objectives, constraints and decision variables to adopt within the optimisation model, a reactor core simulator for function evaluations, and a metaheuristic solver.

Apart from FORMOSA, the tools discussed above provide only single-objective decision support, although ICFM is inherently multi-objective in nature [1]. Examples of conflicting objectives that may be pursued simultaneously are the minimisation of fuel cycle costs, the maximisation of the operational safety of a reactor, and the maximisation of neutron leakage from a research reactor core at its experimental facilities. In multi-objective optimisation (MOO), the aim is to identify a set of trade-off solutions (possibly Pareto optimal) to an optimisation problem. Decision makers should then choose a preferred solution from this set according to their preferences. This is the case in the FORMOSA suite of codes. It is not always obvious, however, how to choose such a preferred solution, and so a decision maker may require additional support. In multiple criteria decision analysis (MCDA), the aim is to identify a preferred solution from a given set of alternatives by incorporating the preferences of a decision maker into the identification process. Accordingly, some level of MCDA support in multi-objective ICFM is crucial for the incorporation of decision maker preferences in order to settle upon a single, final reload configuration decision. To the best of our knowledge, no research into the development or application of MCDA techniques to ICFM is available in the literature.

Another observation that may be made in respect of the aforementioned tools is that they have all been designed for application to power reactors. Decision support for ICFM should, however, also extend to research reactors, since their operators are faced with many of the challenges that power reactor operators also face (and sometimes even more).

In this paper, we attempt to address the shortcomings in the existing ICFM DSS tools, as identified in the discussion above, by proposing a generic optimisation-based DSS framework for multiobjective in-core fuel management (MICFM). In principle, the framework is applicable to any light water power and research reactor for single-cycle MICFM decision support. The conceptualisation of the framework is novel in the sense that, to the best of our knowledge, there are no other decision support frameworks with the aforementioned application scope in the ICFM literature. The framework, then, is intended to serve as a high-level formalisation of a computerised tool that can assist nuclear reactor operators (i.e., the decision makers) in MICFM reload configuration design.

\section{DECISION SUPPORT SYSTEMS}

A decision support system is a computer-based tool or application that can assist a user in complex decision making and problem solving tasks [15]. Essentially, a DSS should be capable of identifying possible decision alternatives, determining their consequences, and providing recommendations following an evaluation of those consequences [16]. These capabilities result in a DSS that supports Herbert Simon's popular decision making model, which is characterised by three phases: intelligence, design, and choice [15-17].

In a classical design proposed by Sprague [17], a DSS consists of three major components: the data subsystem, the model subsystem, and the user interface. This DSS design is presented graphically in Figure 1. The data subsystem has access to external and internal data, using sophisticated database management techniques. The model subsystem consists of different modelling functions that may be used to construct an appropriate decision making model. Lastly, the user interface component 
enables a user of the DSS to interact with the system in an easily-understandable manner. Most importantly, it allows the user to analyse and choose decision alternatives.

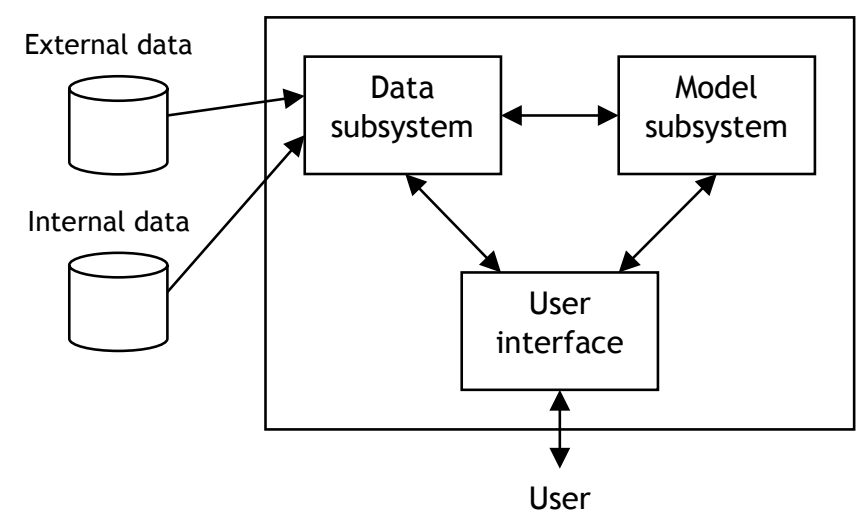

Figure 1: The classical design of a decision support system proposed by Sprague [17]

As mentioned in [15], a considerable volume of research has been conducted in respect of each of the components of this classic DSS. Accordingly, systems have evolved significantly over the years, resulting in a large variety of different types of DSSs today. The interested reader is referred to $[15,18]$ for a history of these developments and a listing of the different DSS types.

A personal DSS, according to Arnott and Pervan [18], is a small-scale system typically developed for one or a small number of independent users, aimed at supporting one decision task. This is an appropriate type of DSS in the context of MICFM because the user is typically a nuclear reactor operator, and the single decision task is the choice of which reload configuration to adopt for a reactor. The prevailing literature reveals that an optimisation model can, to a large extent, capture the intricacies of MICFM $[1,19,20]$. From a technical point of view, it therefore follows - according to Alter's taxonomy of DSSs in [18] - that a model-oriented personal DSS for MICFM based on optimisation models is expected to be a suitable DSS type to pursue. Furthermore, if artificial intelligence techniques are employed for the solution of an optimisation model in the DSS, then the system may also be referred to as an intelligent DSS or a personal DSS embedded with artificial intelligence techniques [18].

\section{THE PROPOSED OPTIMISATION-BASED DSS FRAMEWORK}

A framework for a generic optimisation-based DSS in the context of MICFM is presented in this section. In this framework, the DSS consists of six major components: a database management system (DBMS), a problem generator (PG), an optimisation engine $(\mathrm{OE})$, a function evaluator (FE), an optional auxiliary optimisation system (AOS), and a human-machine interface (HMI). A simple diagrammatic representation of the proposed system is presented in Figure 2.

The DBMS component maintains an internal collection of information and knowledge, apart from retrieving required input data from external sources. In the PG component, a specific MICFM optimisation problem instance (and its corresponding model) is generated, using input and modelling information from the DBMS and/or through the HMI. An MOO solver within the OE component then identifies reload configuration alternatives by solving the optimisation model. This is followed by a level of MCDA support (also within the OE component) in order to facilitate the choice of one reload configuration by the user. In order to evaluate any candidate reload configuration, the FE component is employed. The optional AOS component consists of various supporting features that may enhance the functioning of optimisation in the DSS. Finally, all interactions between a user and the DSS are facilitated by the HMI component.

The components in the DSS framework are intended to be modular in design. This modularity should improve the robustness and general applicability of the DSS, since it allows for components (or subcomponents) to be easily extended, replaced, or even removed entirely. An overview of each component is presented diagrammatically in the following sections, along with a detailed discussion of their functions. In the diagrams, optional DSS sub-components are indicated by dashed lines. 


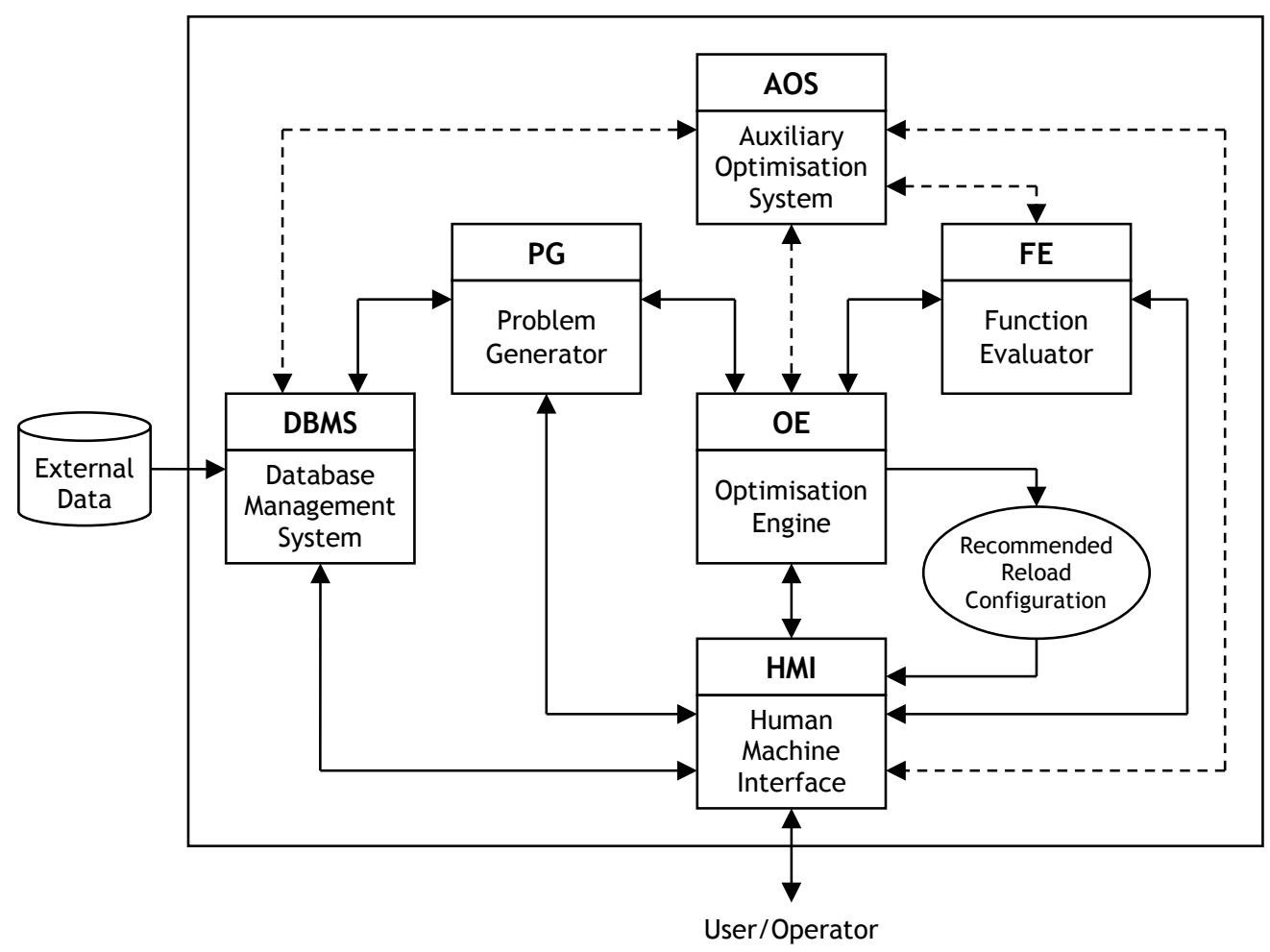

Figure 2: A simple diagrammatic representation of the proposed decision support system

\subsection{The database management system (DBMS)}

The DBMS component is presented in Figure 3. It consists of an internal collection of information and knowledge, along with management routines for access, modification, and retrieval purposes. External data required for MICFM, such as the number and type of fresh fuel assemblies to use, and what the planned reactor cycle length should be, also feed into the DBMS. The external data are primarily sourced from the so-called out-of-core fuel management decision process. This process generally entails making long-term (i.e., multicycle) fuel management decisions $[1,20]$.

The most important part of the DBMS is the fuel assembly inventory in which every fuel assembly that has in the past formed part, or that will form part, of future ICFM decisions for a reactor is tracked. The isotopic composition of any fuel assembly, in particular, has to be known at any given time because of the influence it has on reactor performance.

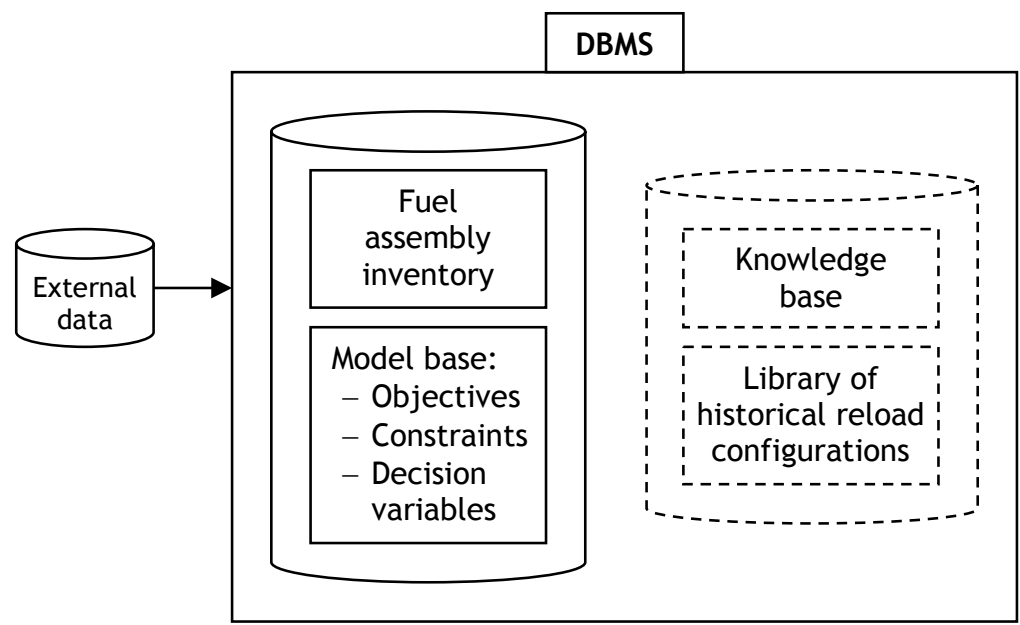

Figure 3: The database management system (DBMS) component 
A model base also forms part of the DBMS and, as mentioned in Section 2, it should contain different modelling functions that may be used to construct an optimisation problem instance and corresponding model. Consistent with the existing DSSs described in the introduction, a variety of known objectives, constraints, and decision variables should be available for the user to choose from. New model elements may, however, also be added by means of the HMI if they do not form part of the current model base.

Vast amounts of experience have been accumulated by experts in the field of ICFM over the years, and this may be included in the DSS to aid in optimisation. This invaluable experience may be captured within a knowledge base as part of the DBMS and may be revised at any stage using the HMI. Similarly, a library of historical reload configurations may form part of the DBMS, and it should contain previous reload configurations known to have performed well. These may be used as starting points, should it be so desired, in the search for new configurations.

\subsection{The problem generator (PG)}

The PG component of the DSS is presented in Figure 4. A specific MICFM optimisation problem instance is generated within the PG. Accordingly, a problem instance sub-component comprises the selected objectives, constraints, and decision variables obtained either from direct user input through the HMI, or from the model base in the DBMS. The fuel assembly data for optimisation is also present in the PG component, and consists of the relevant data (as required in the problem instance) from a subset of the fuel assemblies tracked in the DBMS. This subset corresponds only to those assemblies that should be considered for potential reloading in the reactor core, for the given operational cycle.

Finally, a model translator sub-component is responsible for constructing the optimisation model associated with the optimisation problem instance. This model should be represented in a suitable format for use in the OE component of the DSS - i.e., to enable the application of an MOO solver.

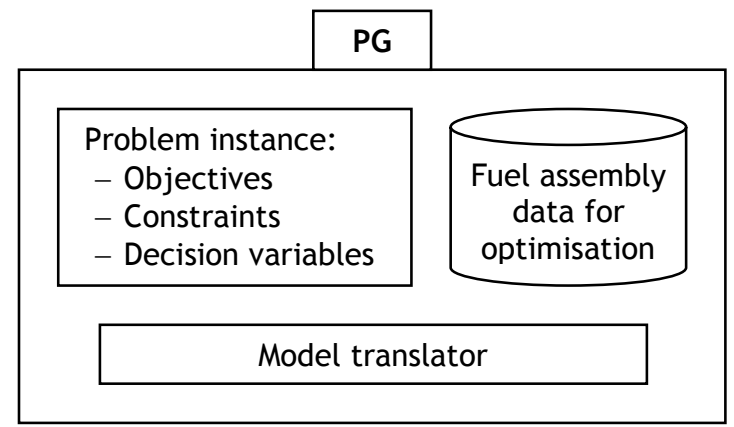

Figure 4: The problem generator (PG) component

\subsection{The optimisation engine (OE)}

The OE component forms the heart of the optimisation-based DSS, and is presented graphically in Figure 5. The primary level of decision support is rendered by a multi-objective optimisation solver sub-component. It is used to solve the optimisation model received from the PG component. The MOO solver may employ an exact or approximate solution technique, while special care may be needed in respect of constraint handling.

A set of trade-off reload configurations (i.e., Pareto optimal or an approximation thereof) is returned by the $\mathrm{MOO}$ solver and forwarded to a multiple criteria decision analysis engine sub-component. At this point, the secondary level of decision support is rendered: the MCDA engine refines the set of configurations by incorporating user preferences until a final reload configuration may be recommended. This recommendation is ultimately the result returned by the DSS.

Depending on the technique employed within the MCDA engine, it may be necessary to explore a promising sub-region of the Pareto set in greater detail. Accordingly, the MOO solver may be invoked again for this purpose. Similarly, if the decision maker rejects the entire set of trade-off reload configurations, the $\mathrm{MOO}$ solver may be employed to re-solve the optimisation model and return a new set of configurations for consideration. 


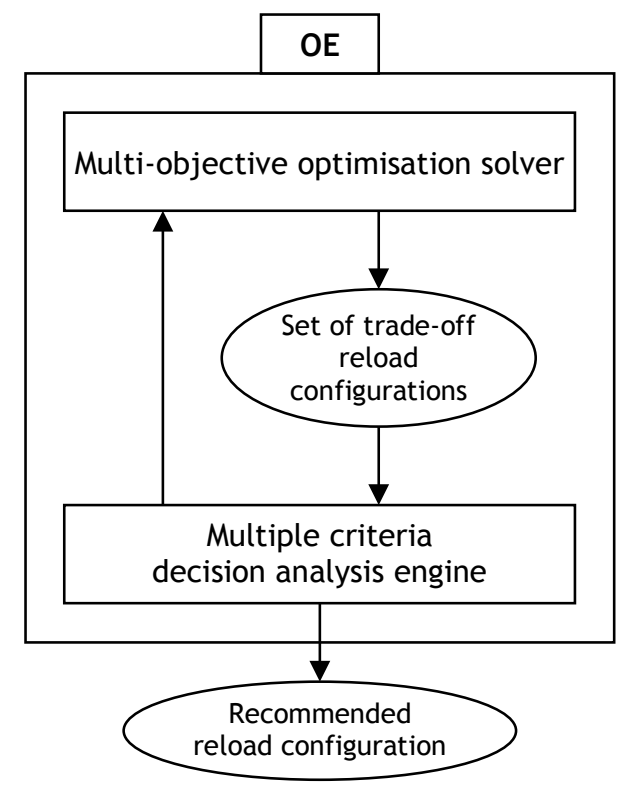

Figure 5: The optimisation engine (OE) component

\subsection{The function evaluator (FE)}

The FE component is used for evaluating the objective functions and constraints of any reload configuration obtained within the $\mathrm{OE}$ component. A detailed overview of the FE component is presented in Figure 6. Central to the FE is a high-fidelity reactor core simulator sub-component that, at the very least, is required to validate the function values of a candidate reload configuration. This validation serves two purposes: it ensures that all the safety-related regulatory requirements for a candidate reload configuration are met, and it yields highly accurate objective function values for the user to consider. Accordingly, the simulator should be applied to the set of trade-off reload configurations returned by the $\mathrm{MOO}$ solver so as to eliminate any invalid configurations from further contention in the MCDA engine. If an excessive number of reload configurations were eliminated in this manner, it may result in the MCDA engine using distorted information and recommending an unsatisfactory reload configuration. The MOO solver may then be employed to re-solve the optimisation model, potentially adopting more stringent limits within the constraints present in the model.

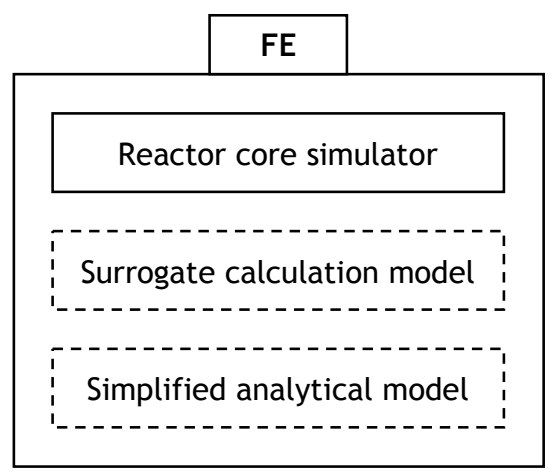

Figure 6: The function evaluator (FE) component

That same core simulator may also be employed to perform the function evaluations during optimisation in the OE component, although such use of the core simulator may be computationally expensive. Accordingly, a computationally cheaper surrogate calculation model sub-component may be used for function evaluations so as to improve the DSS's efficiency. Similarly, a simplified analytical model sub-component may also be employed for the purpose of function evaluations, depending on the optimisation model. 


\subsection{The auxiliary optimisation system (AOS)}

The optional AOS component is presented in Figure 7. It consists of various sub-components that may enhance the functioning of the optimisation process within the DSS. In the solver enhancement features sub-component, a mixed-use function evaluating approach may be adopted in which a surrogate calculation model is used as a screening tool to pre-evaluate reload configurations quickly, after which the reactor core simulator evaluates those configurations that have passed the screening process. Another enhancement feature may be to use the knowledge base in the DBMS in conjunction with the MOO solver to exclude certain regions of the decision space, rather focusing the search in regions known to be promising. The feedback received during or after the application of the $\mathrm{OE}$ engine may be exploited in a self-learning update subsystem that is capable of revising the surrogate model and/or the knowledge base in the DBMS. Also, an optimisation algorithm may be seeded with historical reload configurations from the DBMS to initialise the search with good solutions.

In most optimisation algorithms applied in the context of $\mathrm{MOO}$, tuning parameters have to be selected by the user. The quality of the optimisation results may be sensitive to these values. Inclusion of an automated parameter tuning subsystem in the AOS component may remove the burden of parameter tuning from the user.

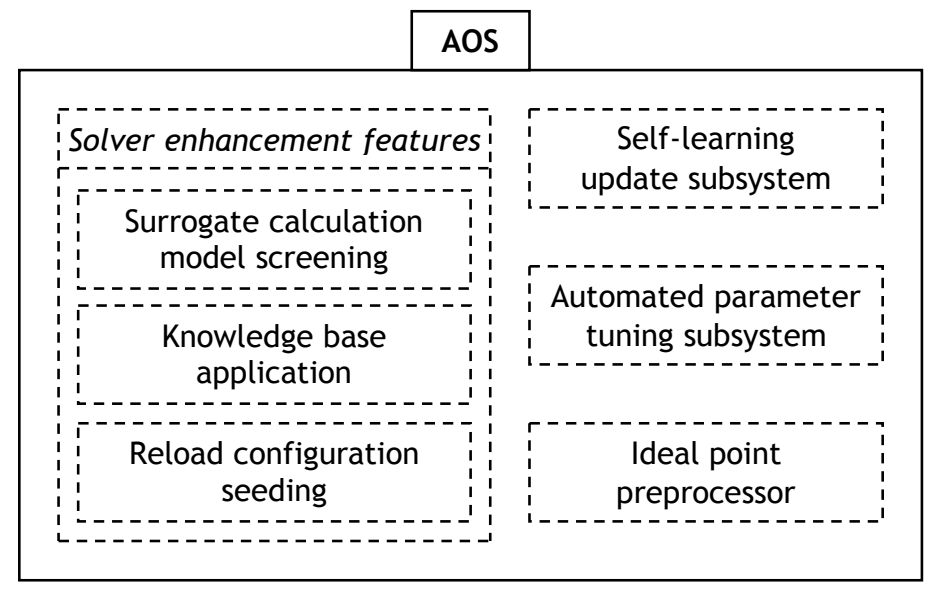

Figure 7: The auxiliary optimisation system (AOS) component

Finally, knowledge of the so-called ideal point [21] (or an approximation thereof) is required in many MCDA techniques. The ideal point is a vector whose elements are obtained by optimising each of the multiple objective functions individually, subject to the same constraints. Very often, the ideal points for practical optimisation problems, such as MICFM, are not known. It may therefore prove useful to the MCDA engine if an ideal point preprocessor sub-component were included in the AOS. In the sub-component, a single-objective optimisation solver may calculate the ideal point, or an approximation thereof.

\subsection{The human-machine interface (HMI)}

As mentioned earlier, the HMI component facilitates all interactions between a user and the DSS. The HMI should preferably be a fully interactive graphical computer interface, easily understood by both technical and non-technical users (e.g., analysts and managers). It is especially crucial that the results obtained by the $M O O$ solver and MCDA engine be presented in an appropriate manner in the HMI: a user should be convinced that the recommended reload configuration suitably captures his/her preferences. Once a reload configuration has been recommended, the HMI should display all the pertinent information about that configuration. A delicate balance should, however, be struck in the design of the $\mathrm{HMI}$ so as to avoid, on the one hand, an information overload (since humans have limited processing capacities [16]) and, on the other hand, excessive automation (since this may weaken a user's understanding and interpretation of the results).

\section{CONCLUSION}

A framework for an optimisation-based DSS in the context of MICFM was proposed in this paper. The DSS in the framework consists of five major components: a database management system, a problem 
generator, an optimisation engine, a function evaluator, an auxiliary optimisation system, and a human-machine interface. A detailed overview of each component was presented. Due to the generic nature of the framework, it should, in principle, be applicable to any light water power and research reactor for single-cycle MICFM decision support. The conceptualisation of the framework is novel in the sense that, to the best of our knowledge, there are no other proposals in the ICFM literature having its application scope. Accordingly, the conceptual framework may serve as the basis for developing a computerised tool that may aid nuclear reactor operators in MICFM reload configuration design.

Potential future work may include populating the components (and sub-components) of the proposed optimisation-based DSS for MICFM with various techniques and approaches in order to demonstrate its functionality. For example, recent studies have shown that multi-objective metaheuristics are very effective at solving the MICFM optimisation problem [22], and may therefore be adopted in the MOO solver sub-component. Similarly, artificial neural networks have been shown to work well as surrogate calculation models [23], and have been applied successfully for screening purposes [24], whereas an extensive knowledge base has been developed for ICFM optimisation in [8]. A particular challenge would be to develop and/or apply appropriate techniques for the MCDA engine subcomponent, given that no such research has been conducted in the context of MICFM as far as the authors are aware.

\section{ACKNOWLEDGEMENTS}

The authors (E.B. Schlünz, P.M. Bokov, and J.H. van Vuuren) were financially supported in part by the National Research Foundation (NRF) of South Africa (Grant 88003, Grant 96281, and Grant 70593 respectively). Any opinion, finding, and conclusion or recommendation expressed in this material is that of the author(s), and the NRF does not accept any liability in this regard.

\section{REFERENCES}

[1] Turinsky, P.J. 2005. Nuclear fuel management optimization: A work in progress, Nuclear Technology, 151, pp 3-8.

[2] Ho, L. \& Rohach, A.F. 1982. Perturbation theory in nuclear fuel management optimization, Nuclear Science and Engineering, 82, pp 151-161.

[3] Hongchun, W. 2001. Pressurized water reactor reloading optimization using genetic algorithms, Annals of Nuclear Energy, 28, pp 1329-1341.

[4] Kropaczek, D.J. \& Turinsky, P.J. 1991. In-core nuclear fuel management optimization for pressurized water reactors utilizing simulated annealing, Nuclear Technology, 95, pp 9-32.

[5] Lin, C. \& Lin, B. 2012. Automatic pressurized water reactor reloading pattern design using ant colony algorithms, Annals of Nuclear Energy, 43, pp 91-98.

[6] Sauar, T.O. 1971. Application of linear programming to in-core fuel management optimization in light water reactors, Nuclear Science and Engineering, 46, pp 274-283.

[7] Alim, F., Ivanov, K. \& Levine, S.H. 2008. New genetic algorithms (GA) to optimize PWR reactors Part I: Loading pattern and burnable poison placement optimization techniques for PWRs, Annals of Nuclear Energy, 35, pp 93-112.

[8] Nissan, E. \& Galperin, A. 1998. Refueling in nuclear engineering: The FUELCON project, Computers in Industry, 37, pp 43-54.

[9] Verhagen, F.C.M., Van der Schaar, M., De Kruijf, W.J.M., Van de Wetering, T.F.H. \& Jones, R.D. 1997. ROSA, a utility tool for loading pattern optimization, Proceedings of the Advances in Nuclear Fuel Management II Conference, Myrtle Beach (SC), USA.

[10] Galperin, A., Kimhi, S. \& Segev, M. 1989. A knowledge-based system for optimization of fuel reload configurations, Nuclear Science and Engineering, 102, pp 43-53.

[11] Nissan, E., Siegelmann, H., Galperin, A. \& Kimhi, S. 1997. Upgrading automation for nuclear fuel in-core management: From the symbolic generation of configurations, to the neural adaptation of heuristics, Engineering with Computers, 13, pp 1-19.

[12] Maldonado, G.I. \& Turinsky, P.J. 1995. Application of nonlinear nodal diffusion generalized perturbation theory to nuclear fuel reload optimization, Nuclear Technology, 110, pp 198-218.

[13] Stevens, J.G., Smith, K.S., Rempe, K.R. \& Downar, T.J. 1995. Optimization of pressurised water reactor shuffling by simulated annealing with heuristics, Nuclear Science and Engineering, 121, pp 67-88.

[14] DeChaine, M.D. \& Feltus, M.A. 1995. Nuclear fuel management optimization using genetic algorithms, Nuclear Technology, 111, pp 109-114.

[15] Shim, J.P., Warkentin, M., Courtney, J.F., Power, D.J., Sharda, R. \& Carlsson, C. 2002. Past, present and future of decision support technology, Decision Support Systems, 33, pp 111-126. 
[16] Pomerol, J. \& Adam, F. 2004. Practical decision making - From the legacy of Herbert Simon to decision support systems, Proceedings of the 2004 IFIP TC8/WG8.3 International Conference: Decision support in an uncertain and complex world, 151, pp 647-657.

[17] Sprague, R.H. 1980. A framework for the development of decision support systems, MIS Quarterly, 4(4), pp 1-26.

[18] Arnott, D. \& Pervan, G. 2005. A critical analysis of decision support systems research, Journal of Information Technology, 20, pp 67-87.

[19] Quist, A.J., Van Geemert, R., Hoogenboom, J.E., Illés, T., Roos, C. \& Terlaky, T. 1999. Application of nonlinear optimization to reactor core fuel reloading, Annals of Nuclear Energy, 26, pp 423-448.

[20] Turinsky, P.J. 2010. Core isotopic depletion and fuel management, in Cacuci D.G. (Ed.), Handbook of Nuclear Engineering, Springer.

[21] Miettinen, K. 1999. Nonlinear multiobjective optimization, Kluwer Academic Publishers.

[22] Schluinz, E.B., Bokov, P.M. \& Van Vuuren, J.H. 2016. A comparative study on multiobjective metaheuristics for solving constrained in-core fuel management optimisation problems, Computers \& Operations Research, 75, pp 174-190.

[23] Schluinz, E.B., Bokov, P.M. \& Van Vuuren, J.H. 2015. Application of artificial neural networks for predicting core parameters for the SAFARI-1 nuclear research reactor, Proceedings of the $44^{\text {th }}$ Annual Conference of the Operations Research Society of South Africa, Hartbeespoort, South Africa, pp 12-22.

[24] Yamamoto, A. 2003. Application of neural network for loading pattern screening of in-core optimization calculations, Nuclear Technology, 144, pp 63-75. 